University of Nebraska - Lincoln

DigitalCommons@University of Nebraska - Lincoln

\title{
Proposed method for measuring the duration of electron pulses by attosecond streaking
}

\author{
Peter Reckenthaeler \\ Max-Planck-Institut für Quantenoptik \\ Martin Centurion \\ University of Nebraska-Lincoln, martin.centurion@unl.edu \\ V. S. Yakovlev \\ Ludwig-Maximilians-Universität München, \\ M. Lezius \\ Max-Planck-Institut für Quantenoptik \\ F. Krausz \\ Max-Planck-Institut für Quantenoptik
}

See next page for additional authors

Follow this and additional works at: https://digitalcommons.unl.edu/physicscenturion

Part of the Physics Commons

Reckenthaeler, Peter; Centurion, Martin; Yakovlev, V. S.; Lezius, M.; Krausz, F.; and Fill, Ernst E., "Proposed method for measuring the duration of electron pulses by attosecond streaking" (2008). Martin Centurion Publications. 8.

https://digitalcommons.unl.edu/physicscenturion/8

This Article is brought to you for free and open access by the Research Papers in Physics and Astronomy at DigitalCommons@University of Nebraska - Lincoln. It has been accepted for inclusion in Martin Centurion Publications by an authorized administrator of DigitalCommons@University of Nebraska - Lincoln. 


\section{Authors}

Peter Reckenthaeler, Martin Centurion, V. S. Yakovlev, M. Lezius, F. Krausz, and Ernst E. Fill 


\title{
Proposed method for measuring the duration of electron pulses by attosecond streaking
}

\author{
P. Reckenthaeler, ${ }^{1,2, *}$ M. Centurion, ${ }^{1}$ V. S. Yakovlev, ${ }^{2}$ M. Lezius, ${ }^{1}$ F. Krausz, ${ }^{1,2}$ and E. E. Fill ${ }^{1}$ \\ ${ }^{1}$ Max-Planck-Institut für Quantenoptik, Hans-Kopfermann-Strasse 1, D-85748 Garching, Germany \\ ${ }^{2}$ Department für Physik der Ludwig-Maximilians-Universität München, Am Coulombwall 1, D-85748 Garching, Germany
}

(Received 27 November 2007; revised manuscript received 11 March 2008; published 7 April 2008)

\begin{abstract}
We propose a method to measure the duration of ultrashort electron pulses. The electron pulse to be measured impinges on a solid target, causing the emission of Auger electrons through impact ionization. The energy spectrum of the Auger electrons is altered in the presence of an intense femtosecond laser field. Due to the extremely short lifetime of the Auger effect, this effect can be used to generate cross correlation between a laser and an electron pulse. The method is applicable to electron pulses ranging from hundreds of attoseconds to hundreds of femtoseconds in duration, and for a few hundreds of electron volts to relativistic energies.
\end{abstract}

DOI: 10.1103/PhysRevA.77.042902

PACS number(s): 79.20.Fv, 78.47.-p, 78.70.En, 79.70.+q

\section{INTRODUCTION}

The quest for ever shorter electron pulses is motivated by a number of exciting applications, including ultrafast electron diffraction, crystallography and microscopy [1-3], electron imaging [4,5], the generation of ultrashort $\mathrm{x}$-ray pulses $[6,7]$, and pumping of $x$-ray lasers [8]. In particular, few fs or even attosecond (as) electron pulses would dramatically advance the technique of time-resolved electron diffraction, permitting the generation of movies of the fastest structural dynamics of molecules and electronic wave packets in atoms [2,9-11]. Femtosecond electron pulses have been generated using a very short electron gun to reduce space-chargeinduced broadening [2] and by laser irradiation of metal nanotips $[12,13]$. Suggestions have been made to produce femtosecond electron pulses by gating with surface plasmons [14] or by ponderomotive deflection [15]. Attosecond electron pulse generation has been proposed by means of highintensity laser pulses [16] and by bunching using a "temporal lens" [17] or a radio-frequency cavity [18].

A key problem in most applications is to measure the duration of ultrashort electron pulses. Several methods have been investigated and applied for this purpose including streak cameras [19], interferometry of coherent transition radiation $[19,20]$, radio-frequency zero phasing [21], terahertz radiation diagnostics [22], electro-optic encoding [23], and ponderomotive interaction of a laser with the electron pulse [24]. None of these methods, however, has proved to be applicable in a wide range of parameters: Streak cameras and radio-frequency zero phasing cannot be used for pulses shorter than a few hundred fs. Coherent transition radiation, as well as terahertz radiation and electro-optic methods, requires a large number of electrons per pulse to provide a sufficient signal, while the ponderomotive interaction requires high laser intensities and is limited by the duration of intense laser pulses.

In this paper, we present a method for measuring the duration of electron pulses, which holds promise for being applicable to an unprecedentedly broad range of parameters: From femtoseconds to attoseconds, from electron volts to

\footnotetext{
*peter.reckenthaeler@mpq.mpg.de
}

mega-electron volts, and from millions of electrons per bunch to single-electron pulses. It is similar to that known as the "attosecond-streak camera" [25,26], an ingenious tool for investigating ultrafast processes. So far, attosecond streaking has been used for measurement of soft X-ray pulses, characterizing laser fields and investigating ultrafast processes in atoms [27-32].

\section{THEORY}

The underlying idea is based on the fact that an electron pulse impinging on a solid target will generate, through impact ionization, a pulse of Auger electrons with duration equal to that of the incident pulse convolved with the duration of the Auger decay. As discussed below, Auger decay can be much faster than the duration of the pulses and is easily accounted for. The energy of an Auger electron created in the presence of a laser field is altered by the field. The energy shift $\Delta U$ depends on the phase $\varphi$ of the electric field $E=E_{0} \sin (\varphi)$ at which the electron is released and the electron initial energy $W_{0}$, according to the classical formula [25]

$$
\Delta U=\sqrt{8 W_{0} U_{p}} \cos (\varphi) \cos (\Theta) .
$$

Here, $\Theta$ is the angle between the velocity of the emitted electrons and the laser polarization and $U_{p}=E_{0}^{2} / 4 \omega_{L}^{2}$ is the ponderomotive potential (here given in atomic units) of the laser field, where $E_{0}$ is the maximum amplitude and $\omega_{L}$ the frequency of the laser field, respectively. Equation (1) applies to linearly polarized laser pulses; it shows that even with relatively low laser intensity (corresponding to a small ponderomotive potential) one can obtain quite appreciable energy shifts, which for $W_{0}>U_{p}$ can be much larger than the laser ponderomotive potential. Calculation of the spectra following a quantum-mechanical approach yields side bands spaced by the laser photon energy, with a maximal energy shift given by Eq. (1). Such spectra have been seen in the so-called laser-assisted Auger decay [33]. The expected spectrum can be calculated within a quantum mechanical model similar to the one describing laser-assisted Auger decay initiated by the absorption of an energetic photon [29,34]. First, we consider only those collisions of electrons with atoms that occurred at a certain moment $t$. Without the streaking 
field, the energy distribution of Auger electrons is given by $[35,36]$

$$
\begin{aligned}
\widetilde{I}(U, t) \propto \frac{1}{\sqrt{U}} \mid \int_{t}^{\infty} M(\sqrt{2 U}) \\
\times\left.\exp \left[-\int_{t}^{t^{\prime}}\left(\frac{\Gamma}{2}+i\left(U-W_{0}\right)\right) d \tau\right] d t^{\prime}\right|^{2},
\end{aligned}
$$

where $\Gamma$ is the rate of the Auger decay and $M(p)$ is the bound-continuum coupling matrix element as a function of the free-electron momentum $p=\sqrt{2 U}$. This model neglects the post-collision interactions, as well as the interaction of the Auger electron with the field created by the electron pulse. We also assume here that the collision moment $t$ is well defined, which is a valid assumption as long as the interaction time between a projectile and an atom is much smaller than the Auger decay time $1 / \Gamma$. Under these assumptions, the interaction of a free electron with a homogeneous time-dependent streaking field can be described as [26]

$$
\begin{aligned}
\widetilde{I}(U, t) \propto & \frac{1}{\sqrt{U}} \mid \int_{t}^{\infty} M\left(\sqrt{2 U}+A\left(t^{\prime}\right)\right) \exp \left[-\frac{\Gamma}{2}\left(t^{\prime}-t\right)-i W_{0} t^{\prime}\right. \\
& \left.+\frac{i}{2} \int_{0}^{t}[\sqrt{2 U}+A(\tau)]^{2} d \tau\right]\left.d t^{\prime}\right|^{2} .
\end{aligned}
$$

Here, $A(\tau)$ is defined as

$$
A(\tau)=\int_{\tau}^{\infty} E\left(\tau^{\prime}\right) d \tau^{\prime}
$$

and we assume that the interaction of the streaking field with bound electrons does not affect the Auger spectrum.

So far, we have been considering a single electron hitting an atom at a moment $t$. To evaluate the Auger spectrum formed by a pulse of electrons with a finite duration, we assume that the probability to initiate the Auger decay in a given atom is proportional to the instantaneous flux in the electron pulse $I_{e}(t)$. Denoting the relative delay between the laser and the electron pulses as $\Delta t$, we obtain the final expression for Auger spectra dressed by the streaking field,

$$
I(U, \Delta t) \propto \int_{-\infty}^{\infty} I_{e}\left(t-\Delta t^{\prime}\right) \tilde{I}(U, t) d t .
$$

Different atoms-hit by different electrons-emit Auger wave packets independently. Therefore, deriving Eq. (5), we incoherently added contributions from atoms that experienced collisions at different moments.

\section{PROPOSED EXPERIMENT}

We propose to use the change in the Auger line in the presence of the laser field for measuring electron pulse durations in an arrangement conceptually shown in Fig. 1: The ultrashort electron pulse impinges on a suitable material generating Auger electrons. A short laser pulse is then focused at grazing incidence on the surface of the solid, its polarization direction being coincident with the direction in which the

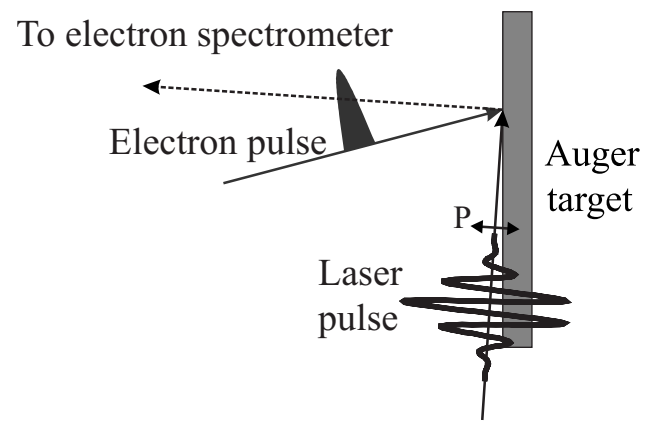

FIG. 1. Basic arrangement of electron pulse duration measurement. $\mathrm{P}$ indicates the direction of polarization of the laser pulse.

Auger electrons escaping from the target are detected. An appropriate choice of the angles of the electron and laser beams with respect to the surface prevents a deterioration of the temporal resolution due to geometrical effects. The angles must be chosen such that the relative delay between the pulses is constant along the target surface; for example, for relativistic electrons, a collinear geometry is optimal, while for $20 \mathrm{keV}$ electrons the optimum angle is approximately $15^{\circ}$ with respect to the surface normal (for grazing incidence of the laser). A time-of-flight electron spectrometer then records electron spectra for different time delays between laser and electron pulses. Overlaying a sufficient number of spectra with different delays generates a "streaked" spectrum from which the electron pulse duration can be deduced.

\section{NUMERICAL RESULTS}

In the following, we present results of the calculations for two different cases, viz., relatively long laser and electron pulses (of order $100 \mathrm{fs}$ ), and very short pulses, viz., a fewcycle laser pulse with an as electron pulse. These very different parameter ranges illustrate the broad applicability of the method. In the long-pulse case (electron pulse duration longer than the laser cycle), the electron spectrum at any particular delay will be broadened, since the electron energies are swept through a large number of laser cycles. In the short-pulse regime (electron pulse duration shorter than laser cycle), the electron spectrum will be broadened and shifted. Note that an Auger line with an appropriate decay time must be chosen for optimum results in the two regimes. The spectrum is independent of the energy of the incident electron pulse. The energy does, however, affect the cross section for generating Auger electrons, as discussed below in more detail.

The following parameters were used for the calculation of the long-pulse case: Electron pulse duration $100 \mathrm{fs}$, laser pulse duration $50 \mathrm{fs}$. Both pulses were assumed to be Gaussian in time. For the Auger transition, the oxygen $K L L$ line with an energy of $500 \mathrm{eV}$ is chosen. The natural width of this line is $0.15 \mathrm{eV}$ corresponding to a decay time of $4.4 \mathrm{fs}$. The laser intensity is assumed to be $1.6 \times 10^{12} \mathrm{~W} / \mathrm{cm}^{2}$ at a wavelength of $800 \mathrm{~nm}$, corresponding to a ponderomotive potential $U_{p}=0.1 \mathrm{eV}$. The maximum energy shift, calculated from Eq. (1), is $\pm 20 \mathrm{eV}$. Figure 2 shows the oxygen line and its 


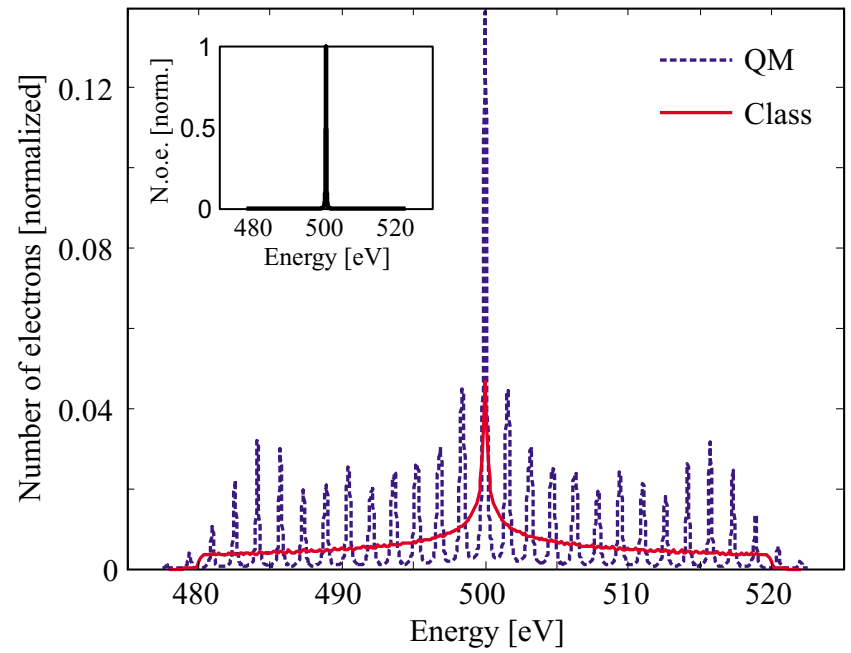

FIG. 2. (Color online) Electron spectra for a long-pulse case at delay time $\Delta t=0$, i.e., when electron and laser pulse maxima coincide. The dashed blue curve (QM) shows the result of a quantum mechanical calculation following Eq. (5), while the red solid curve (Class) shows the spectrum calculated (classically) using Eq. (1). The inset shows the original line without laser. The total number of electrons is the same for all curves.

broadening calculated using Eqs. (1) and (5). The boundcontinuum transition matrix element $M$ was assumed to be constant within the bandwidth of interest.

The electron spectrum is seen to be substantially altered during the period in which the two pulses overlap: According to the quantum mechanical formula (5) the intensity of the main Auger line is reduced to $14 \%$ and side bands spaced by the photon energy appear on both sides, extending up to the maximum energy shift, while the classical calculation predicts a decrease of the main peak to $5 \%$, along with a continuous broadening of the line. Even though the main Auger line is still quite prominent in the spectrum, measurement of the number of the energy-shifted electrons should be readily feasible, yielding the cross-correlation signal between the envelopes of the laser and the electron pulses. Apart from the expected sidebands, the quantum mechanical approach also shows a modulation in the amplitude of the sidebands. The modulation arises from interference between different components of the electron wave packet emitted with the same energy at the rising and falling part of the laser cycle. The streaked spectrum, generated by plotting the spectra as functions of delay between laser and electron pulse, is shown in Fig. 3(a). As the overlap between the two pulses increases, more and more sidebands appear on both sides of the main Auger line. From such a correlation measurement, the duration of the electron pulse can easily be retrieved if the laser pulse envelope is known.

The short-pulse case [Fig. 3(b)] is calculated with the following parameters: Electron pulse duration 500 as, a fewcycle cosine-laser pulse with duration of $4 \mathrm{fs}$ at a wavelength of $800 \mathrm{~nm}$. Again, a laser intensity of $1.6 \times 10^{12} \mathrm{~W} / \mathrm{cm}^{2}$, i.e., $U_{p}=0.1 \mathrm{eV}$, was assumed. Note that the laser pulse must be carrier-envelope stabilized, otherwise the temporal resolution would only be given by its pulse duration. For the Auger transition, the titanium KLL Auger line at $4.06 \mathrm{keV}$ with a

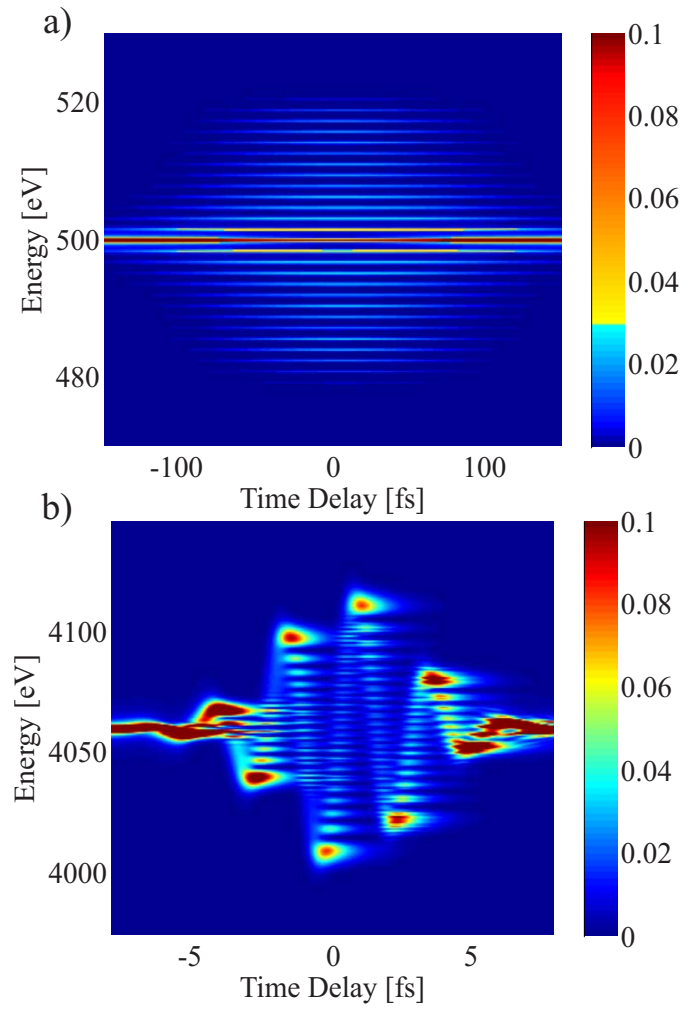

FIG. 3. (Color online) Calculated electron spectra. (a) Spectrum for long-pulse conditions. Electron pulse duration $100 \mathrm{fs}$; laser pulse duration $50 \mathrm{fs}$. Auger line applied oxygen $K L L$ at $500 \mathrm{eV}$. (b) Spectrum for short-pulse conditions as a function of time delay. Electron pulse duration 500 as; laser pulse duration 5 fs. Auger line Ti $K L L$ at $4.06 \mathrm{keV}$. Time zero is defined as the temporal delay at which both pulse maxima coincide. The color scale is normalized to the intensity of the original Auger line.

natural width of $0.94 \mathrm{eV}$ and, correspondingly, a decay time of 702 as is chosen. Equation (1) predicts for the maximum energy shift of the electrons a value of $\pm 57 \mathrm{eV}$. Features quite different from the long-pulse case are now observed: The main change in the spectrum is an energy shift which occurs in synchronism with the laser vector potential. Slight broadening results from the combined smearing of electron pulse duration and Auger decay time over the laser cycle.

For successful realization of the experiment, the number of Auger electrons generated must be high enough to produce a spectrum in an appreciable amount of time. The electron yield $\eta_{\mathrm{el}}$, defined as the number of Auger electrons per pulse electron can be calculated by the expression $\eta_{\mathrm{el}}$ $=\left(1-\eta_{f}\right) \sigma_{K} N_{0} d_{\text {esc }}$, where $\eta_{f}$ is the fluorescence yield of the Auger line, $\sigma_{k}$ is the cross section for $K$-hole generation by the incoming electrons, $N_{0}$ is the number density of the solid, and $d_{\mathrm{esc}}$ is the escape depth of the Auger electrons from the solid. The fluorescence yields for oxygen and titanium are given by 0.0083 and 0.214 , respectively [37]. For nonrelativistic electrons, the calculated cross section for generating a $K$ hole are $2.6 \times 10^{-20} \mathrm{~cm}^{2}$ and $1.06 \times 10^{-21} \mathrm{~cm}^{2}$ for oxygen and titanium, respectively [38]. The escape depth can be taken from the universal mean-free-path curve for solids (see, for example, [39]), and is $0.9 \mathrm{~nm}$ at $500 \mathrm{eV}$ and about $3 \mathrm{~nm}$ at $4 \mathrm{keV}$. Using these parameters we obtain $\eta_{\mathrm{el}, \mathrm{O}}$ 
$=1.7 \times 10^{-4}$ and $\eta_{\mathrm{el}, \mathrm{Ti}}=1.4 \times 10^{-5}$. For the calculation of $\eta_{\mathrm{el}, \mathrm{O}}$, the number density of oxygen in sapphire was used. These numbers show that with an electron gun running at 1000 electrons per pulse at a $\mathrm{kHz}$ repetition rate or with 1 electron per pulse at a $\mathrm{MHz}$ repetition rate, and an electron spectrometer with an acceptance angle of about 1 sterad, a complete spectrum covering a range of $W_{0} \pm \Delta U$ can be recorded in less than an hour (although for cross-correlation experiment a smaller range could be sufficient).

In order for the method to work over a wide range of electron energies, the cross section for generating a $K$ hole must be sufficiently large. In the energy range between $\mathrm{keV}$ and $\mathrm{GeV}$, the cross section has a minimum around $\mathrm{MeV}$ energies $[40,41]$. Even at the lowest value the cross section is comparable to the value used in the above example, thus we expect the method to work from $\mathrm{keV}$ to $\mathrm{GeV}$ energies. The minimum energy for the electron pulse is given by the energy necessary to create the $K$ hole (just slightly above the energy of the chosen Auger line). We have also considered the effect of the finite escape depth, but do not expect it to be significant, given that the escape time (over 1 mean free path) will be below 100 as.

\section{CONCLUSION}

In conclusion, we present a method of electron pulse duration measurement. By drawing on laser assisted Auger electron emission induced by impact ionization, the method can be used for determining electron pulse durations in a broad range of parameters of electron energy and pulse duration. Electron energies may range from a few $\mathrm{keV}$ up to highly relativistic ones in the $\mathrm{GeV}$ range and pulse durations from a few 100 fs to sub fs. The method also works independently of the number of electrons per pulse.

\section{ACKNOWLEDGMENTS}

This work was funded in part by DFG under Contract No. SFB Transregio 6039 and by the DFG Cluster of Excellence "Munich Centre for Advanced Photonics"-MAP (www.munich-photonics.de). M.C. is supported by the Alexander von Humboldt Foundation. P.R. is supported by the International Max Planck Research School on Advanced Photon Science-IMPRS-APS (www.mpq.mpg.de/APS).
[1] A. H. Zewail, Annu. Rev. Phys. Chem. 57, 65 (2006).

[2] J. R. Dwyer, C. T. Hebeisen, R. Ernstorfer, M. Harb, V. B. Deyirmenjian, R. E. Jordan, and R. J. Dwayne Miller, Philos. Trans. R. Soc. London, Ser. A 364, 741 (2006).

[3] V. A. Lobastov, R. Srinivasan, and A. H. Zewail, Proc. Natl. Acad. Sci. U.S.A. 102, 7069 (2007).

[4] Y. Okano, Y. Hironaka, K. G. Nakamura, and K. Kondo, Appl. Phys. Lett. 83, 1536 (2003).

[5] C. G. Serbanescu and R. Fedosejevs, Appl. Phys. B: Lasers Opt. 83, 521 (2006).

[6] H. Schwoerer, B. Liesfeld, H.-P. Schlenvoigt, K.-U. Amthor, and R. Sauerbrey, Phys. Rev. Lett. 96, 014802 (2006).

[7] R. Schoenlein, W. Leemans, A. Chin, P. Volfbeyn, T. Glover, P. Balling, M. Zolotorev, K. Kim, S. Chattopadhyay, and C. Shank, Science 274, 236 (1996).

[8] D. Kim, C. Toth, and C. P. J. Barty, Phys. Rev. A 59, R4129 (1999).

[9] J. Itatani, J. Levesque, D. Zeidler, N. Niikura, H. Pepin, J. C. Kieffer, P. B. Corkum, and D. M. Villeneuve, Nature (London) 432, 867 (2004).

[10] H. Niikura, F. Legare, R. Hasbani, A. D. Bandrauk, M. Y. Ivanov, D. M. Villeneuve, and P. B. Corkum, Nature (London) 417, 917 (2002).

[11] P. B. Corkum and F. Krausz, Nat. Phys. 3, 381 (2007).

[12] P. Hommelhoff, C. Kealhofer, and M. A. Kasevich, Phys. Rev. Lett. 97, 247402 (2006).

[13] C. Ropers, D. R. Solli, C. P. Schulz, C. Lienau, and T. Elsaesser, Phys. Rev. Lett. 98, 043907 (2007).

[14] S. E. Irvine and A. Y. Elezzabi, Opt. Express 14, 4115 (2006).

[15] P. Baum and A. H. Zewail, Proc. Natl. Acad. Sci. U.S.A. 103, 16105 (2006).

[16] N. Naumova, I. Sokolov, J. Nees, A. Maksimchuk, V. Yanovsky, and G. Mourou, Phys. Rev. Lett. 93, 195003
(2004).

[17] M. Monastyrskiy, S. Andreev, D. Greenfield, G. Bryukhnevich, V. Tarasov, and M. Schelev, in High-Speed Photography and Photonics, edited by B. J. Thompson (SPIE, 2005), Vol. 5580, p. 324.

[18] E. Fill, L. Veisz, A. Apolonski, and F. Krausz, New J. Phys. 8, 272 (2006).

[19] T. Watanabe, M. Uesaka, J. Sugahara, T. Ueda, K. Yoshii, Y. Shibata, F. Sakai, S. Kondo, M. Kando, H. Kotaki, and K. Nakajima, Nucl. Instrum. Methods Phys. Res. A 437, 1 (1999).

[20] H. C. Lihn, P. Kung, C. Settakorn, H. Wiedemann, and D. Bocek, Phys. Rev. E 53, 6413 (1996).

[21] D. X. Wang, G. A. Krafft, and C. K. Sinclair, Phys. Rev. E 57, 2283 (1998).

[22] J. van Tilborg, C. B. Schroeder, C. V. Filip, C. Toth, C. G. R. Geddes, G. Fubiani, E. Esarey, and W. P. Leemans, Phys. Plasmas 13, 056704 (2006).

[23] I. Wilke, A. M. MacLeod, W. A. Gillespie, G. Berden, G. M. H. Knippels, and A. F. G. van der Meer, Phys. Rev. Lett. 88, 124801 (2002)

[24] C. T. Hebeisen, R. Ernstorfer, M. Harb, T. Dartigalongue, R. E. Jordan, and R. J. Dwayne Miller, Opt. Lett. 31, 3517 (2006).

[25] J. Itatani, F. Quere, G. L. Yudin, M. Y. Ivanov, F. Krausz, and P. B. Corkum, Phys. Rev. Lett. 88, 173903 (2002).

[26] M. Kitzler, N. Milosevic, A. Scrinzi, F. Krausz, and T. Brabec, Phys. Rev. Lett. 88, 173904 (2002).

[27] R. Kienberger, E. Goulielmakis, M. Uiberacker, A. Baltuska, V. Yakovlev, F. Bammer, A. Scrinzi, T. Westerwalbesloh, U. Kleineberg, U. Heinzmann, M. Drescher, and F. Krausz, Nature (London) 427, 817 (2004).

[28] M. Hentschel, R. Kienberger, C. Spielmann, G. A. Reider, N. Milosevic, T. Brabec, P. Corkum, U. Heinzmann, M. Drescher, 
and F. Krausz, Nature (London) 414, 509 (2001).

[29] M. Drescher, M. Hentschel, R. Kienberger, M. Uiberacker, V. Yakovlev, A. Scrinzi, T. Westerwalbesloh, U. Kleineberg, U. Heinzmann, and F. Krausz, Nature (London) 419, 803 (2002).

[30] E. Goulielmakis, M. Uiberacker, R. Kienberger, A. Baltuska, V. Yakovlev, A. Scrinzi, T. Westerwalbesloh, U. Kleineberg, U. Heinzmann, M. Drescher, and F. Krausz, Science 305, 1267 (2004).

[31] A. L. Cavalieri, N. Müller, T. Uphues, V. Yakovlev, A. Baltuska, B. Horvath, B. Schmidt, L. Blümel, R. Holzwarth, S. Hendel, M. Drescher, U. Kleineberg, P. M. Echenique, R. Kienberger, and F. Krausz, Nature (London) 449, 1029 (2007).

[32] G. Sansone, E. Benedetti, F. Calegari, C. Vozzi, L. Avaldi, R. Flammini, L. Poletto, P. Villoresi, C. Altucci, R. Velotta, F. Stagira, S. De Silvestri, and M. Nisoli, Science 314, 443 (2006).
[33] J. M. Schins, P. Breger, P. Agostini, R. C. Constantinescu, H. G. Muller, G. Grillon, A. Antonetti, and A. Mysyrowicz, Phys. Rev. Lett. 73, 2180 (1994).

[34] O. Smirnova, V. S. Yakovlev, and A. Scrinzi, Phys. Rev. Lett. 91, 253001 (2003).

[35] U. Fano, Phys. Rev. 124, 1866 (1961).

[36] R. O. Barrachina and J. H. Macek, J. Phys. B 22, 2151 (1989).

[37] M. O. Krause, J. Phys. Chem. Ref. Data 8, 307 (1979).

[38] D. B. Brown, in Handbook of Spectroscopy, edited by J. W. Robinson (CRC Press, Inc., Boca Raton, FL, 1979), Vol. I, p. 248.

[39] A. Zangwill, Physics at Surfaces (Cambridge University Press, Cambridge, 1990).

[40] J. H. Scofield, Phys. Rev. A 18, 963 (1978).

[41] D. H. H. Hoffmann, C. Brendel, H. Genz, W. Löw, S. Müller, and A. Richter, Z. Phys. A 293, 187 (1979). 